\title{
CFD Analysis of Passive Autocatalytic Recombiner
}

\author{
B. Gera, P. K. Sharma, R. K. Singh, and K. K. Vaze \\ Reactor Safety Division, Bhabha Atomic Research Centre, Trombay, Mumbai 400085, India \\ Correspondence should be addressed to B. Gera, bgera@barc.gov.in
}

Received 10 May 2011; Revised 27 July 2011; Accepted 27 July 2011

Academic Editor: Iztok Tiselj

Copyright (C) 2011 B. Gera et al. This is an open access article distributed under the Creative Commons Attribution License, which permits unrestricted use, distribution, and reproduction in any medium, provided the original work is properly cited.

\begin{abstract}
In water-cooled nuclear power reactors, significant quantities of hydrogen could be produced following a postulated loss-ofcoolant accident (LOCA) along with nonavailability of emergency core cooling system (ECCS). Passive autocatalytic recombiners (PAR) are implemented in the containment of water-cooled power reactors to mitigate the risk of hydrogen combustion. In the presence of hydrogen with available oxygen, a catalytic reaction occurs spontaneously at the catalyst surfaces below conventional ignition concentration limits and temperature and even in presence of steam. Heat of reaction produces natural convection flow through the enclosure and promotes mixing in the containment. For the assessment of the PAR performance in terms of maximum temperature of catalyst surface and outlet hydrogen concentration an in-house 3D CFD model has been developed. The code has been used to study the mechanism of catalytic recombination and has been tested for two literature-quoted experiments.
\end{abstract}

\section{Introduction}

Within the nuclear industry, passive autocatalytic recombiners (PARs) are installed in advanced water-cooled power reactor containments as a combustible gas control system during severe accident conditions. During a severe accident in a nuclear power plant, the high-temperature zirconium fuel cladding can react with high-temperature steam to produce hydrogen. Hydrogen thus generated has the potential to cause uncontrolled combustion in the containment building, which represents a threat to the integrity of the confinement due to pressure and temperature levels. Numerous techniques have been developed for mitigating the potential for such accidents. A number of strategies for mitigating this potential have been adopted. Preinerting, postaccident inerting, electrical recombiner, catalytic recombiner, igniters, and mixing by use of fans are several methods to mitigate the hydrogen hazards in nuclear containments. The one based on passive catalytic recombination of $\mathrm{H}_{2}$ with $\mathrm{O}_{2}$ in air appears to be the most promising one [1]. Autocatalytic recombiners are passive; that is, they are self-starting and self-feeding; they have no moving parts and require no external energization. They come into action spontaneously as soon as the hydrogen concentration begins to increase in the atmosphere. The gas mixture flows through the recombiner by natural convection, pushed by the gas heated as a result of the reaction. These natural convective flow currents promote mixing of combustible gases in the containment.

Most commonly PARs use platinum or palladium as the catalyst as they have the ability to adsorb hydrogen and oxygen. The recombination reaction occurs spontaneously at the catalyst surfaces, and the heat of reaction produces a natural convection flow through the enclosure. Water vapor as a product of reaction along with remaining hydrogenair mixture moves upward because of buoyancy, and fresh hydrogen-air mixture enters through the bottom inlet section. However, limited conversion capacities that may not be sufficient at high hydrogen release rates or overheating of the catalyst elements caused by strong reaction heat generation that may lead to unintended ignition of the gaseous mixture are known important issues for improving today's systems. However, the present lack of detailed knowledge with regard to the processes inside a passive autocatalytic recombiner makes any optimization effort difficult. A profound understanding of the processes inside a recombiner, such as reaction kinetics or heat and mass transfer requires detailed investigation of a small recombiner section under well-defined steady-state conditions. Under controlled and conservative conditions the function of the recombiner sheets is assessed by means of the temperature measured on the catalyst sheets and the remaining hydrogen concentration at the outlet of the test device. 
Various experimental and theoretical investigations have been performed worldwide to evaluate and improve the performance of PARs. Several analytical models have been developed to study the recombiner behaviour. Avakian and Braillard [2] have developed an analytical model for catalytic recombiner in a closed vessel and validated this with experimental data from KALI test facility. Tahara et al. [3] have used the STAR-CD code for 3-D modeling of recombination in Battelle Model Containment. Heitsch [4] has used the CFX code to study the detailed fluid dynamics for a separate effect recombiner test used in Battelle Model Containment. Dharwadkar [5] and Prabhudharwadkar et al. [6] have used the FLUENT code for modeling hydrogen distribution along with catalytic recombination in Indian pressurised heavy water reactor (IPHWR) containment. Goyal [7] has also developed analytical model for predicting the performance of the recombiner in HYMIS (hydrogen mitigation studies) test facility.

To provide optimal and reliable conditions and to understand the internal processes inside the recombiner, modeling the device in detail is required. This requirement suggested making use of computational fluid dynamics techniques to simulate hydrogen recombiners, aiming at a multidimensional analysis of the reaction zone with the use of discretization of the relevant balance equations. For the analysis of the processes inside a passive autocatalytic recombiner such as reaction kinetics or heat and mass transfer a 3D in-house CFD code has been developed. The code calculates the catalyst temperature and the concentration regression along the catalyst plates. The code has been validated against REKO experiment [8] and has been used to predict maximum catalyst surface temperature and recombiner outlet hydrogen concentration. The code has been utilized to simulate recombiner device used in the Gxtest series at the Battelle Model Containment (B-MC) [4] and REKO test facility [9]. The paper briefly describes some of the details of the experiments, salient features of the CFD numerical model, and results of the studies carried out for the recombiner device.

\section{Physical Problem}

CFD computations have been carried out for two recombiner geometries. These tests have been chosen because of their simplified geometry and easily available results from open literature. The simplified geometry for the computation is shown in Figure 1. The dimensions used for the computation and other parameters are mentioned in next subsection.

2.1. REKO Test Facility. This test facility consists of a vertical flow channel with a rectangular cross section $46 \mathrm{~mm}$ wide $(W)$ and $146 \mathrm{~mm}$ deep. The channel is $504 \mathrm{~mm}$ high $\left(L_{1}+\right.$ $L_{2}+L_{3}$ ) with about $180 \mathrm{~mm}$ of channel length above the sheets $\left(L_{3}\right)$. For the current experiments four sheets made of stainless steel and coated with wash coat/platinum catalyst material were arranged in parallel inside the flow channel. The plates used were $1.5 \mathrm{~mm}$ thick and $143 \mathrm{~mm}$ high $\left(L_{2}\right)$. In the experimental setup they were arranged in parallel with a separation of $8.5 \mathrm{~mm}$. The catalyst elements were exposed to

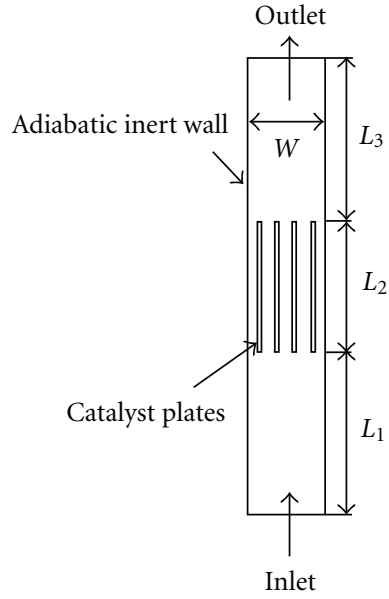

Figure 1: Simplified geometry for CFD computation.

a constant flow of a mixture of air, hydrogen, and/or steam. The hydrogen conversion efficiency was determined from gas analysis measurements during experiments. The catalyst sheets were equipped with thermocouples for measuring the distribution of the catalyst temperature. Experiments have been performed for different flow velocities $(0.25,0.50$, and $0.80 \mathrm{~m} / \mathrm{s})$ at different inlet temperatures $(298,343$, and $383 \mathrm{~K})$. Inlet hydrogen concentrations were varied between 0.5 and $4 \% \mathrm{v} / \mathrm{v}$. Few experiments were also conducted with steam injection at inlet.

2.2. Gx-Test Series at the Battelle-Model Containment (B$M C$ ). This test facility consists of a vertical flow channel with a rectangular cross section $116 \mathrm{~mm}$ wide $(W)$ and $160 \mathrm{~mm}$ deep. The channel is $1600 \mathrm{~mm}$ high $\left(L_{1}+L_{2}+L_{3}\right)$ with about $740 \mathrm{~mm}$ of channel length above the sheets $\left(L_{3}\right)$ to enhance natural convection of the hot exhaust gas. During the experiments 16 sheets made of stainless steel and coated with wash coat/platinum catalyst material were arranged in parallel inside the flow channel. The plates used were $0.5 \mathrm{~mm}$ thick and $150 \mathrm{~mm}$ high $\left(L_{2}\right)$. In the experimental setup they were arranged in parallel with a separation of $10 \mathrm{~mm}$. Temperatures, hydrogen mole fractions, and local velocities were measured at inlet and exit of the recombiner box. During the experiments steady-state conditions were achieved and recorded for basic validation. Two conditions under equilibrium conditions were identified: one with a hydrogen mole fraction of $2.93 \%(\mathrm{Gx} 6,30 \% \mathrm{v} / \mathrm{v}$ steam) and the other with a value of hydrogen mole fraction of $8 \%(\mathrm{Gx} 8.1,60 \% \mathrm{v} / \mathrm{v}$ steam). At the recombiner inlet region mixture temperature, hydrogen mole fraction and velocity were measured. At the outlet temperature and hydrogen mole fraction were recorded. But, along the sheets only surface temperature was measured at two locations.

Experiments at REKO test facility were carried out under forced flow condition and controlled injection. The inlet hydrogen concentration and mixture temperature were less compared to realistic accident condition. The facility has generated useful data for validation of CFD code since detailed measurement was possible. On the other hand, in 
experiments at B-MC test facility recombiner was placed in containment atmosphere. The flow through recombiner was due to natural convection, and steady states were achieved. The inlet hydrogen concentration was high but measurements were made only at few locations.

\section{Mathematical Model}

Detailed analysis of passive autocatalytic recombiner is required to explore the interactions of reaction kinetics, temperature distribution, heat, and mass transfer as well as thermal hydraulic phenomena. The investigation was concentrated on heat generation and its removal and thus maximum temperature obtained by the catalyst plate. In the present CFD model, the interaction of reaction kinetics, heat transfer and associated fluid flow in the catalytic recombiner, and radiation heat loss from plates to surroundings has been studied. The simplified geometry shown in Figure 1 has been modeled by cartesian structured mesh. The governing mass, momentum, energy, and conservation equations for hydrogen, oxygen, nitrogen, and water vapor have been solved in $3 \mathrm{D}$ space. The governing equations are as follows.

Continuity:

$$
\nabla \cdot u=0
$$

Momentum:

$$
(\nabla \cdot \rho u u)=-\nabla p-\left[\nabla \cdot\left(\tau_{i, j}\right)\right]+\rho g,
$$

where

$$
\tau_{i, j}=-\mu\left(\frac{\partial u_{j}}{\partial x_{i}}+\frac{\partial u_{i}}{\partial x_{j}}\right)
$$

Energy:

$$
\nabla \cdot(\rho C p T u)=\nabla \cdot(k \nabla T)+S_{T} .
$$

Species:

$$
\nabla \cdot\left(\rho Y_{i} u\right)=\nabla \cdot\left(\rho D \nabla Y_{i}\right)+S_{y_{i}}
$$

Surface reaction takes places at catalytic plate. This reaction can be modeled by detailed reaction mechanism as mentioned by Deutschmann and another one proposed by Kasemo both reported by [10]. The interest was focused on heat transfer not on the detailed chemical reaction. Thus the reaction has been modeled as a one-step reaction mechanism as proposed by [11]

$$
\mathrm{H}_{2}+\frac{1}{2} \mathrm{O}_{2}=\mathrm{H}_{2} \mathrm{O}
$$

with following reaction rate

$$
\text { Rate }=14 * \exp \left(-\frac{14.9 * 10^{6}}{R_{u} T}\right) *\left[\mathrm{H}_{2}\right] \mathrm{kmol} / \mathrm{m}^{2} / \mathrm{s} .
$$

These reaction mechanisms are specific to experiment. Above reaction rate which is valid for temperature ranges from $450 \mathrm{~K}$ to $1200 \mathrm{~K}$ and is suitable for present work. The above reaction rate has been modelled as the additional source term for energy and water vapor and sinks term for hydrogen and oxygen transport equation. All the source or sink terms were applied in the very first fluid cell adjacent to solid plate. Flow speeds are in the range of $0.25-0.8 \mathrm{~m} / \mathrm{s}$ resulting in Reynolds number $(V L / \nu)$ ranging from 2000 to 6400 . The characteristic length is the length of the reactive plate. The corresponding Grashof number $\left(L^{3} \beta g \Delta T\right) / \nu^{2}$ as a characteristic of free convection heat transfer is approximately $6.0 \times 10^{7}$. Both values are very low and suggest that the flow is essentially laminar. The high energy output from the hydrogen oxygen reaction results in a considerable heat transmission from surfaces by convection and radiation to environment. Heat transfer by radiation from plate to surrounding has not been modelled in the present work. However to model radiation losses a fixed fraction of energy, that is, $30 \%$, has been considered as lost to surroundings (recombiner box and inlet and outlet boundary) from catalyst plates. During simulation for REKO test facility, at the inlet known value of velocity, temperature and species mass fraction have been applied. Outflow boundary condition was used at the outlet while for $\mathrm{Gx}$-test series simulation inlet and outlet were specified as atmospheric pressure boundaries where flow rate was calculated based on mass conservation. Pressure boundaries impose zero normal gradients on temperature and species mass fractions at outlet. At the inlet region the measured inlet values of temperature and species mass fractions were specified. Other boundary conditions were same for both the simulations. Reacting plates have been modelled as conducting walls to model the conjugate heat transfer in solid plates. It was also assumed that water produced by the oxidation reaction is always remaining in vapour form. Hence condensation on the walls has not been modelled. The external wall has been modelled as adiabatic no-slip wall. The governing equations were discretized using Finite Volume Method. Equations were solved for 3D configuration under steady-state condition. The mixture density has been modeled as ideal gas law. A uniform grid was used in all the direction, and staggered scheme of solution was adopted in which fluid velocities were calculated at cell face, and scalars such as pressure, temperature, and species mass fraction were calculated at cell center. The governing equations were iteratively solved with Gauss Seidel method with Patankar's SIMPLE algorithm [12]. Power law scheme was utilized as differencing scheme for the convective terms for all equations, whereas the central difference scheme was used for the diffusive terms.

\section{Results and Discussion}

In this context of detailed analysis of PAR the maximum temperature of catalyst surface and outlet hydrogen concentration has been evaluated. Before performing the parametric study, a grid size sensitivity study was carried out in order to obtain the mesh size for mesh-independent results. The 


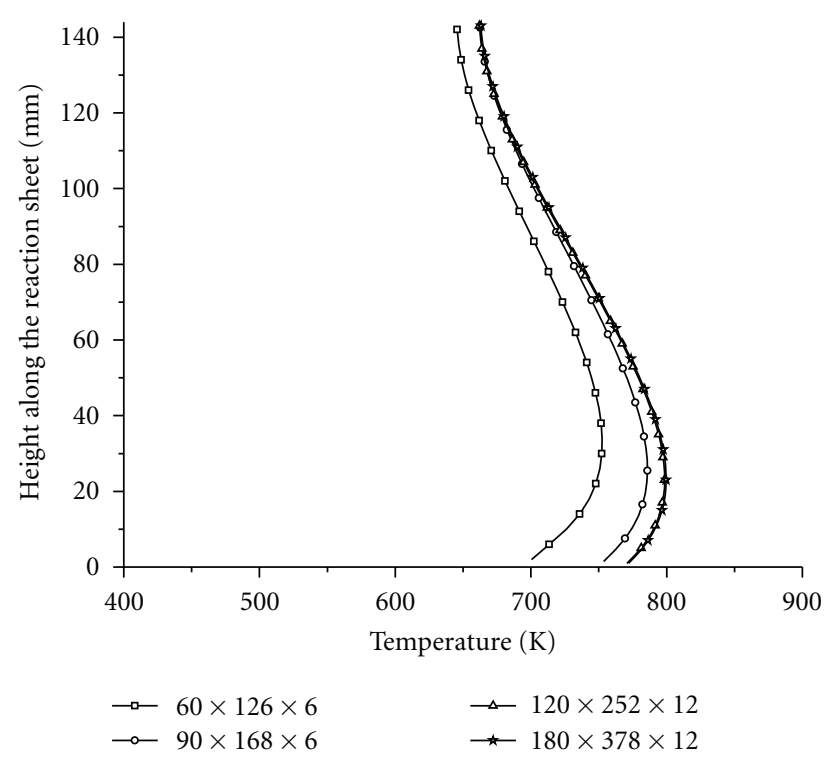

FIGURE 2: The mesh size sensitivity exercise results for REKO test facility simulation $\left(V_{\text {in }}=0.8 \mathrm{~m} / \mathrm{s}, T_{\text {in }}=298 \mathrm{~K}, X \mathrm{H}_{2 \text { in }}=4 \%\right)$.

grid was selected based on grid sensitivity study carried out with $60 \times 126 \times 6,90 \times 168 \times 6,120 \times 252 \times 12$, and 180 $\times 378 \times 12$ grids for geometry of REKO test facility. The results of grid-independent study are depicted in Figure 2 where variation of catalyst plate temperature along the plate height is compared for various grids for a typical case. The calculated temperature varied by about $1 \%$ between the 120 $\times 252 \times 12$ grids and $180 \times 378 \times 12$ grids. Hence for all the simulation for REKO test facility the domain $45 \times 504 \times$ $146 \mathrm{~mm}$ was divided in $120 \times 252 \times 12$ grids which give a grid size of $0.375 \times 2 \times 12.167 \mathrm{~mm}$.

For Gx-test series simulation the domain $160 \times 1485$ $\times 116 \mathrm{~mm}$ was divided in $640 \times 750 \times 10$ grids which give a grid size of $0.25 \times 1.98 \times 11.6 \mathrm{~mm}$. The grid size was decided based on our previous study carried out for REKO experiment facility and experience based on other parametric studies. The code has been validated with available data from the experiments conducted at the REKO-3 test facility. Figures 3 and 4 show the results of the computation carried out for validation purpose. The temperature of the catalyst plate and hydrogen concentration in the recombiner section was obtained with CFD code and compared with experimental results $[9,13]$. In this case hydrogen-air mixture enters at a velocity of $0.8 \mathrm{~m} / \mathrm{s}$ at $343 \mathrm{~K}$ with inlet hydrogen concentration 2 and $4 \%$ v/v. Figure 3 shows the steady-state average hydrogen concentration in the recombiner section comparison of CFD results with experimental results. Figure 4 shows the catalyst surface temperature along the catalytic sheet (central plate) comparison of CFD results with experimental results. The results of present computation are in good agreement with available data.

The analyses were further carried out for inlet temperature 298 and $383 \mathrm{~K}$, with inlet velocity $0.25,0.5$, and $0.8 \mathrm{~m} / \mathrm{s}$ and variation of inlet hydrogen concentration from $1-4 \%$

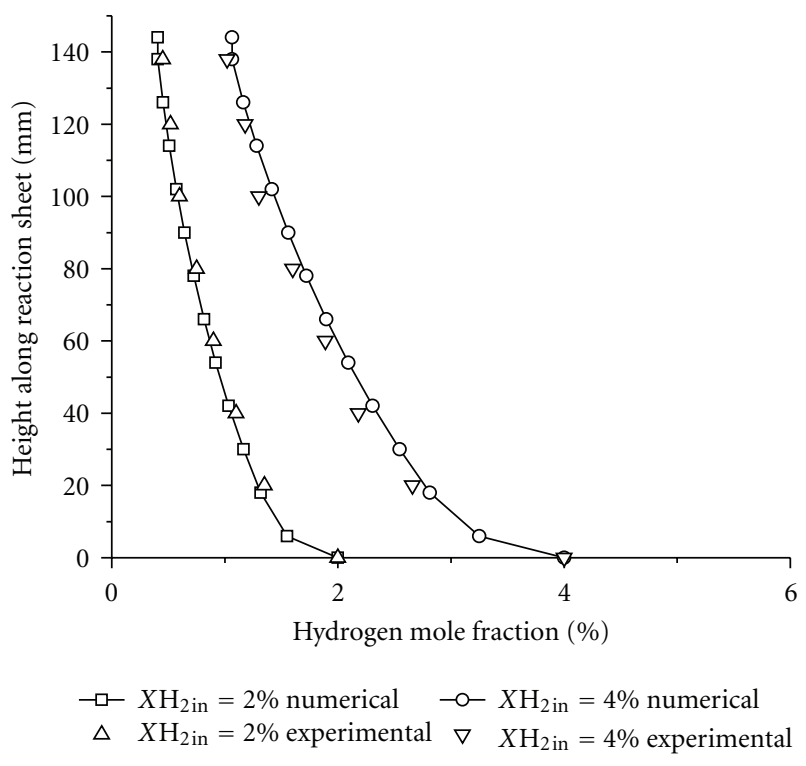

Figure 3: Steady-state average hydrogen concentration in the recombiner section for REKO facility, comparison of CFD results with experimental results for inlet hydrogen mole fraction $2 \%$ \& $4 \%$ and temperature $343 \mathrm{~K}$.

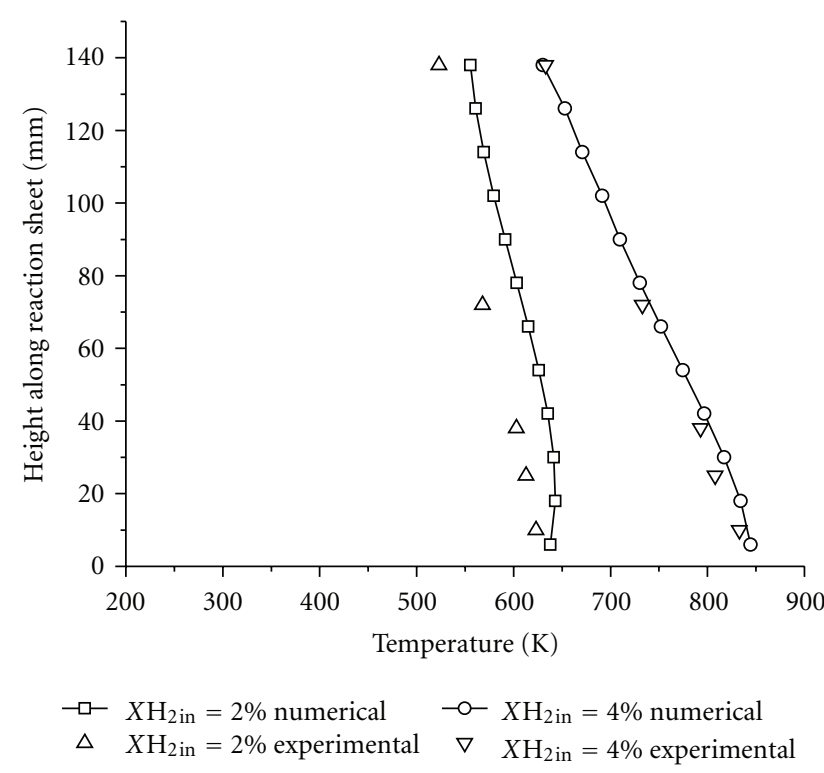

FIGURE 4: Catalyst surface temperature along the catalytic sheet (central plate) for REKO facility, comparison of CFD results with experimental results for inlet hydrogen mole fraction $2 \%$ \& $4 \%$ and temperature $343 \mathrm{~K}$.

v/v for REKO facility, and two analysis were carried out for steady-state conditions one with inlet temperature $355 \mathrm{~K}$ and hydrogen mole fraction of $2.93 \%$ (Gx6, 30\% v/v steam) and the second with inlet temperature $373 \mathrm{~K}$ and a value of hydrogen mole fraction of $8 \%$ (Gx8.1, 60\% v/v steam) for $\mathrm{Gx}$-test series at the (B-MC). Figures 5 and 6 show the temperature and hydrogen concentration for air hydrogen mixture of $2 \% \mathrm{v} / \mathrm{v}$ hydrogen concentration entering at $383 \mathrm{~K}$ 


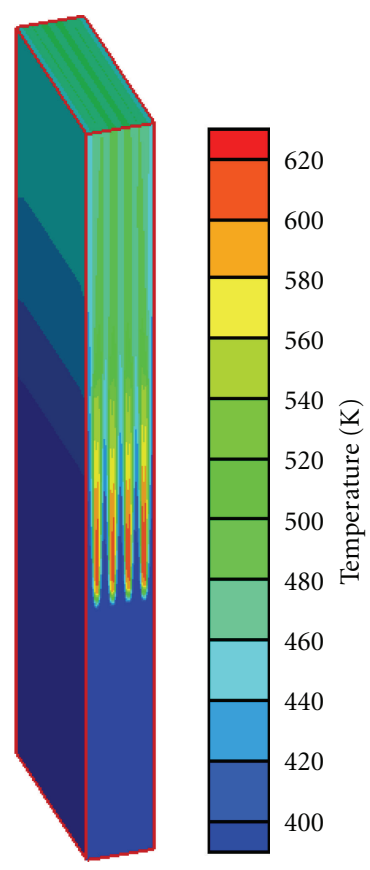

FIGURE 5: Temperature contour for simulation performed for REKO facility for inlet hydrogen mole fraction $2 \%$ and temperature $383 \mathrm{~K}$.

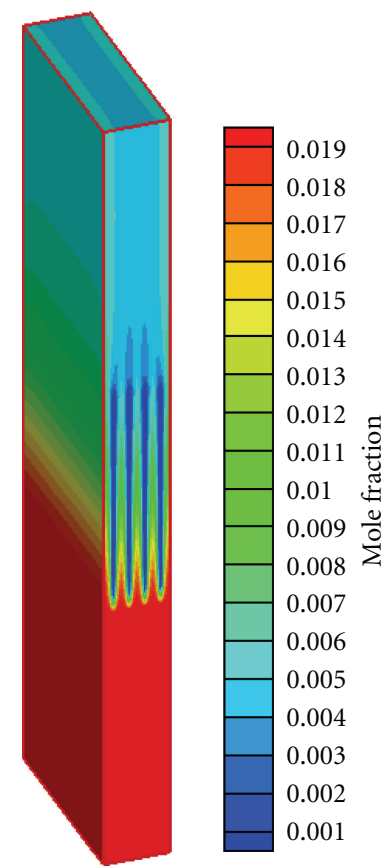

Figure 6: Hydrogen mole fraction contour for simulation performed for REKO facility for inlet hydrogen mole fraction $2 \%$ and temperature $383 \mathrm{~K}$.

for REKO test facility. Figures 7 and 8 show the numerical results for Gx-6 experiment. Figures 9 and 10 depict the maximum catalyst surface temperature as a function of inlet velocity at different inlet hydrogen concentration for inlet mixture temperature 298 and $383 \mathrm{~K}$, respectively, for REKO test facility. As the velocity increases, reaction rate increases

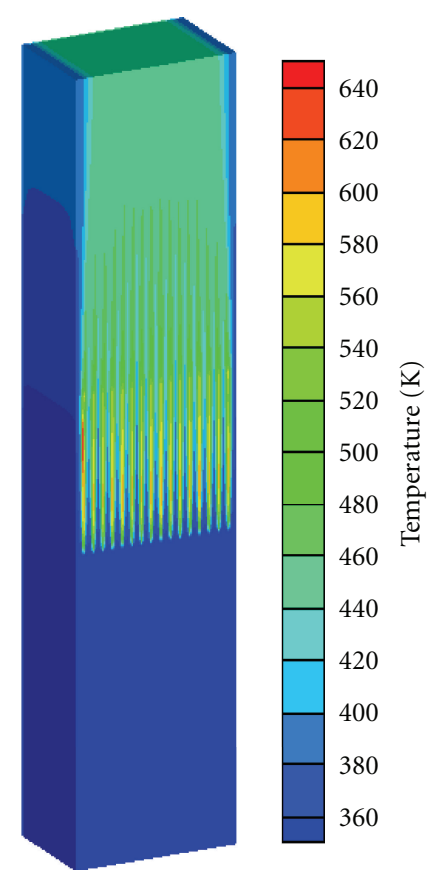

Figure 7: Temperature contour for simulation performed for Gxtest series $(\mathrm{Gx}-6)$.

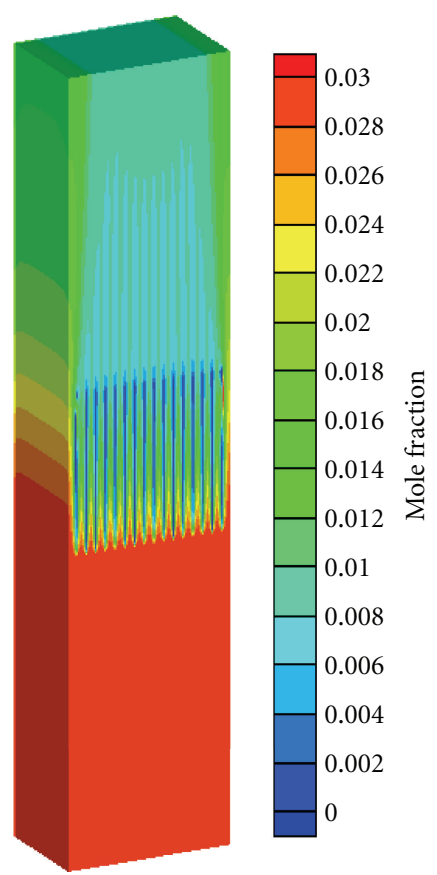

Figure 8: Hydrogen mole fraction contour for simulation performed for Gx-test series (Gx-6).

as more mass enters into the recombiner section as a result temperature of catalytic surface increases. Inlet temperature of mixture also plays some role, and reaction rate is more as inlet mixture temperature is high; correspondingly the catalyst surface temperature is high as the inlet temperature increases from 298 to $383 \mathrm{~K}$. However in both the cases 


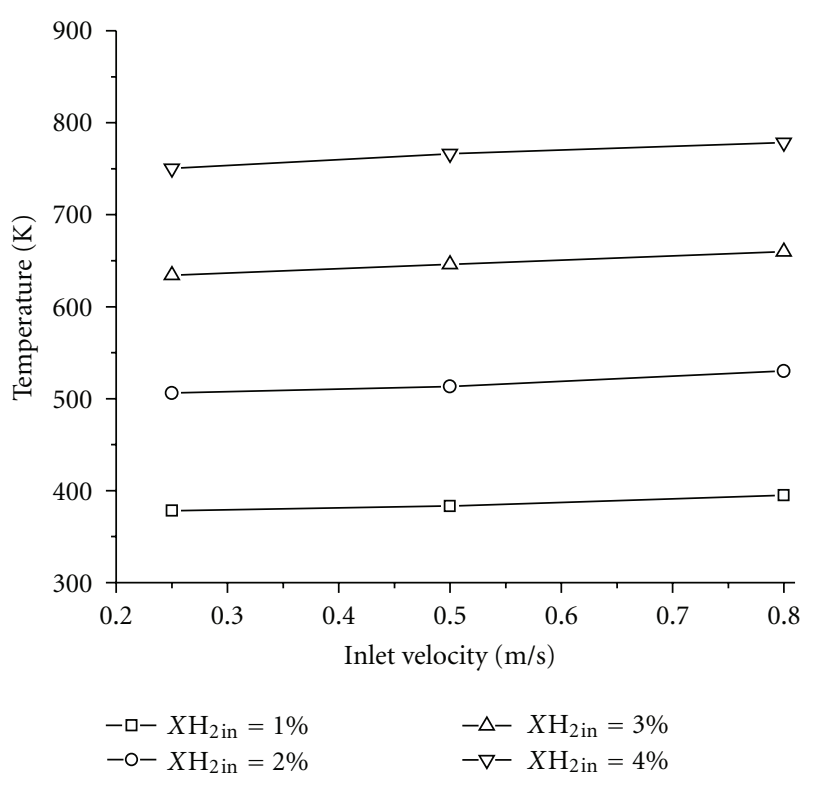

FIGURE 9: Maximum catalyst surface temperature as a function of inlet velocity and inlet hydrogen concentration for REKO facility with inlet temperature $298 \mathrm{~K}$.

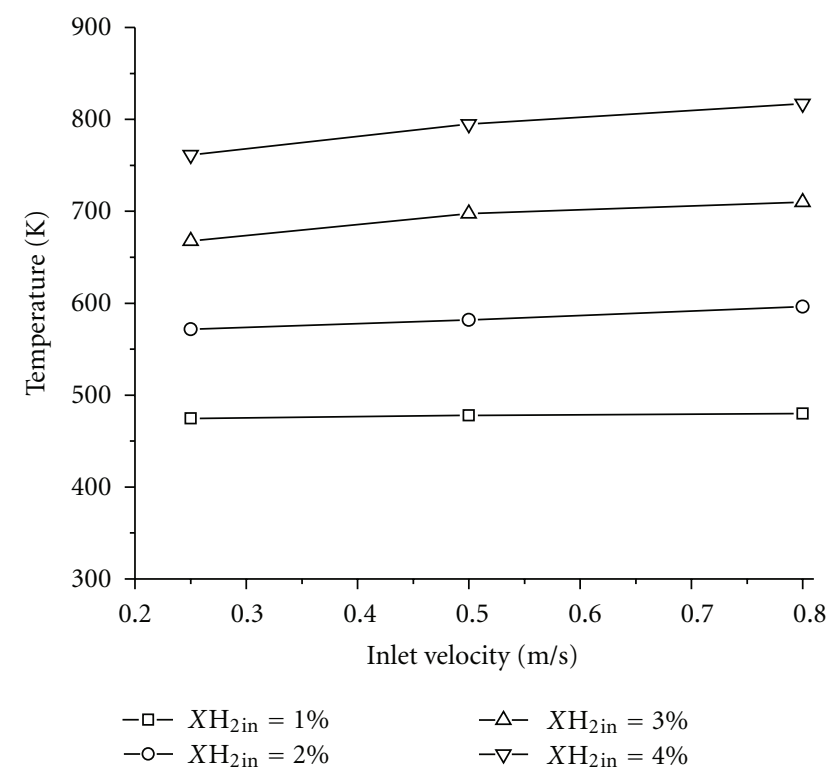

FIGURE 10: Maximum catalyst surface temperature as a function of inlet velocity and inlet hydrogen concentration for REKO facility with inlet temperature $383 \mathrm{~K}$.

variation of maximum catalyst temperature with inlet velocity is small. The catalyst surface temperature is maximum near the leading edge of the plate. As the mixture enters in the recombiner section the reaction occurs at the leading edge of the catalyst sheet. The reaction rate is highest at the leading edge of the plate. This is manifested by sharp decrease in hydrogen concentration and maximum catalyst surface temperature near the leading edge of the plate. The

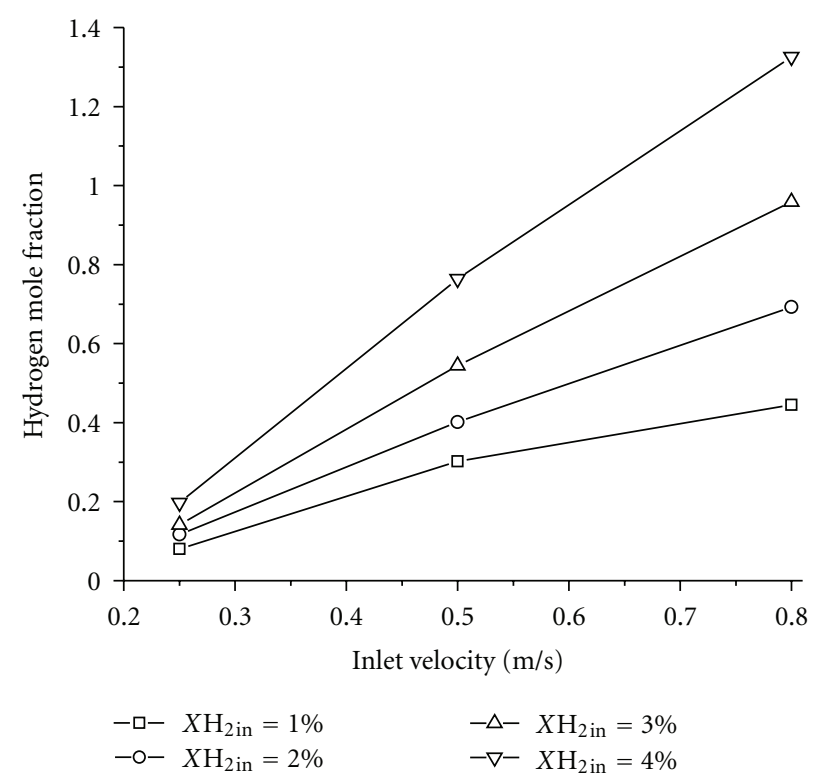

FIGURE 11: Outlet hydrogen concentration as a function of inlet velocity and inlet hydrogen concentration for REKO facility with inlet temperature $298 \mathrm{~K}$.

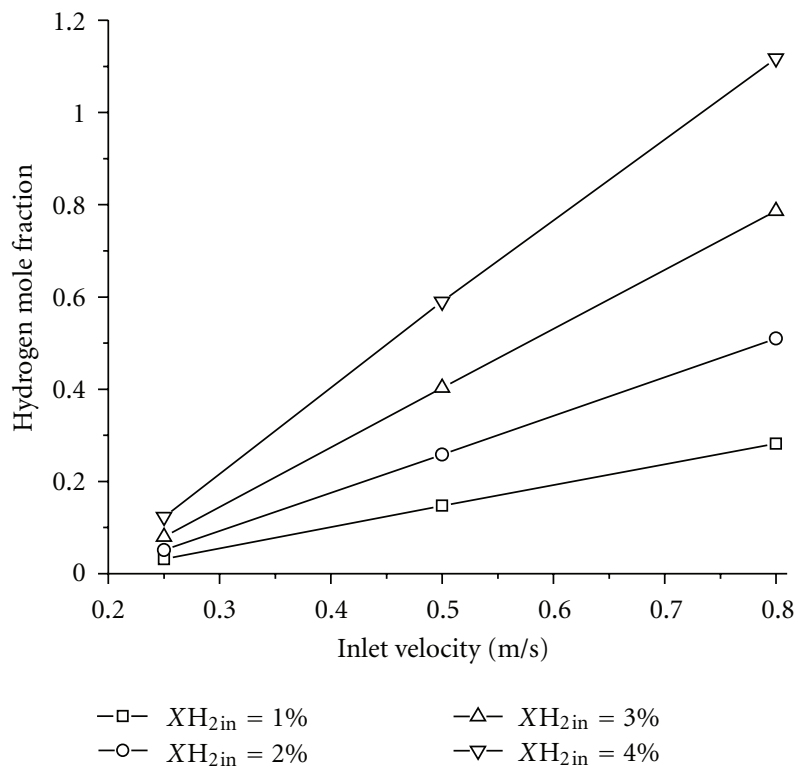

FIGURE 12: Outlet hydrogen concentration as a function of inlet velocity and inlet hydrogen concentration for REKO facility with inlet temperature $383 \mathrm{~K}$.

temperature of plate at trailing edge is high because of heat conduction and convective heat transport. As flow takes place over catalytic plate, boundary layer is formed over the plate surface and hydrogen diffuses from bulk of the mixture towards plates for recombination. As flow takes place along the catalyst sheet concentration gradient decreases thus reaction rate also decreases along the sheet. Both reaction kinetics and diffusional mass transport phenomenon control 


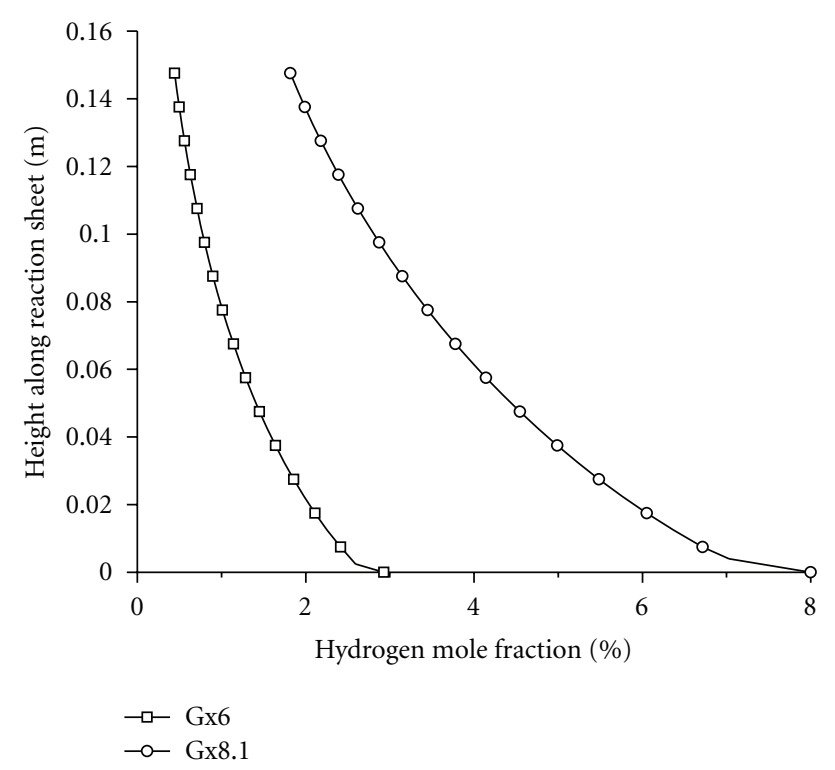

FIGURE 13: Steady-state average hydrogen concentration in recombiner for Gx test series.

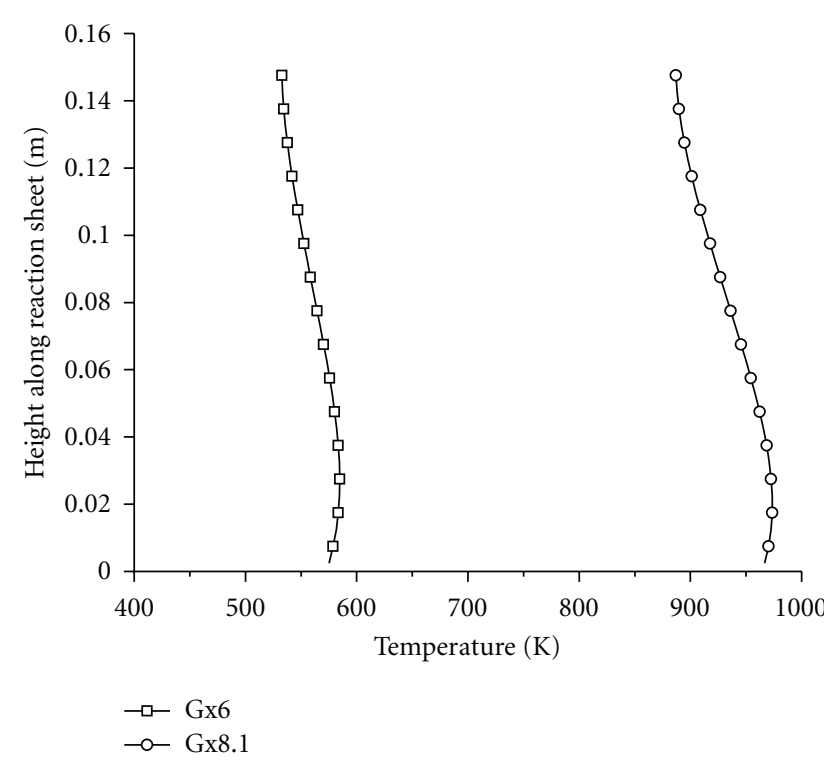

FIGURE 14: Catalyst surface temperatures along the catalytic sheet for Gx test series.

the recombination. Figures 11 and 12 depict the outlet hydrogen concentration as a function of inlet velocity at different inlet hydrogen concentration for inlet mixture temperature 298 and $383 \mathrm{~K}$, respectively, for REKO test facility. The outlet hydrogen concentration depends strongly on inlet speed, lowering the speed the mixture remain in the recombiner for more duration; hence hydrogen consumption is more. Outlet hydrogen concentration is low as the inlet temperature increases from 298 to $383 \mathrm{~K}$. At low speed outlet hydrogen concentration becomes almost zero at both the inlet temperature. These results are consistent with

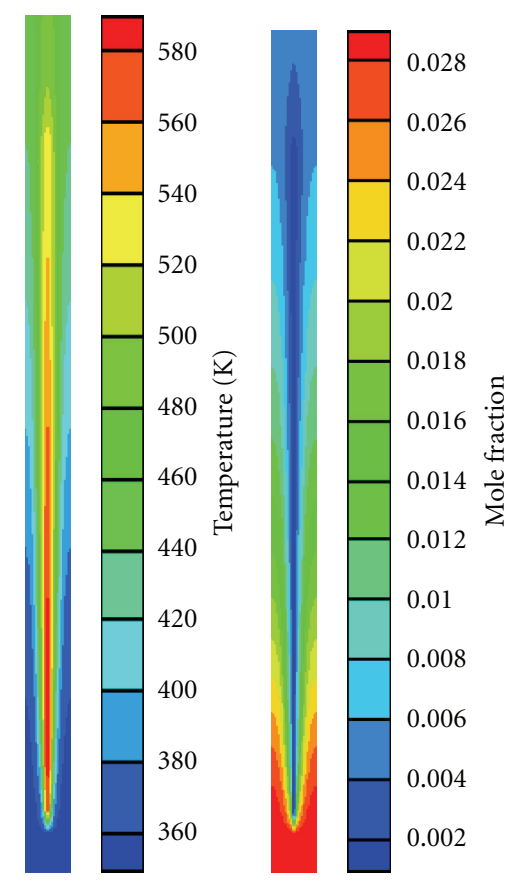

(a)

(b)

FIGURE 15: (a) Temperature contour and (b) hydrogen mole fraction contour zoomed view near catalyst plate for first steadystate condition (Gx-6).

the results obtained by Reinecke et al. [13]. In the central channels reaction takes place on both side of the channel, and boundary layer is formed and merges after a particular height. While in side channels since no reaction occurs at recombiner wall the boundary layer does not merge in this channel. The hydrogen removal rate is higher in central channel while removal rate is lower in side channel; this is clear from the contour plot of hydrogen mole fraction which shows that hydrogen mole fraction is higher near catalytic recombiner box wall. Similarly temperature of the plates in central channel is slightly higher than plates placed near recombiner box wall.

Figure 13 shows the steady-state average hydrogen concentration in recombiner along the height for the analysis carried out for Gx-test series. Figure 14 shows the steadystate catalyst surface temperature along the height for Gxtest series. Figures 15(a) and 15(b) show the zoomed view of temperature and hydrogen concentration near catalyst plate for first steady-state condition ( $\mathrm{Gx}-6)$. Similarly Figures $16(\mathrm{a})$ and $16(\mathrm{~b})$ show the zoomed view of temperature and hydrogen concentration near catalyst plate for second steady-state condition $(\mathrm{Gx}-8.1)$. The shape of hydrogen concentration boundary layer is different in both the cases. For Gx-6 experiment bulk hydrogen concentration is low giving a small driving force for hydrogen to move towards catalyst plate as compared to $\mathrm{Gx}-8.1$ experiment where bulk hydrogen concentration is high. As a result of this thickness of hydrogen concentration boundary layer is more along the reaction sheet for Gx-6 experiment as compared to Gx8.1 experiment. This program has been utilized to predict 
TABLE 1: Comparison of results for first steady-state condition (At inlet mixture temperature $355 \mathrm{~K}$, mole fraction of hydrogen $2.93 \%$, and mole fraction of steam $30 \%$ steam) for Gx-test series.

\begin{tabular}{lccc}
\hline & Experiment & Reported CFX results [8] & Present results \\
\hline Maximum surface temperature (K) & $632 \mathrm{~K}$ & $620 \mathrm{~K}$ & $584 \mathrm{~K}$ \\
Outlet gas temperature $(\mathrm{K})$ & $513 \mathrm{~K}$ & $502 \mathrm{~K}$ & $470 \mathrm{~K}$ \\
Outlet hydrogen mole fraction $(\%)$ & 0.4 & 0.4 & 0.44 \\
\hline
\end{tabular}

TABLE 2: Comparison of results for second steady-state condition (At inlet mixture temperature $373 \mathrm{~K}$, mole fraction of hydrogen $8 \%$, and mole fraction of steam $60 \%$ steam) for Gx-test series.

\begin{tabular}{lccc}
\hline & Experiment & Reported CFX results [8] & Present results \\
\hline Maximum surface temperature (K) & $750 \mathrm{~K}$ & $965 \mathrm{~K}$ & $973 \mathrm{~K}$ \\
Outlet gas temperature (K) & $635 \mathrm{~K}$ & $735 \mathrm{~K}$ & $723 \mathrm{~K}$ \\
Outlet hydrogen mole fraction $(\%)$ & 1.5 & 0.6 & 1.8 \\
\hline
\end{tabular}

the maximum catalyst surface temperature, gas temperature at the recombiner outlet, and hydrogen concentration at recombiner outlet for both the cases of Gx-test series. The present results and comparison with reported experiment and numerical results are tabulated in Tables 1 and 2. In the simulation there is a good match with experimental values. The situation with the lower hydrogen mole fraction (Gx6) is well matched. With $8 \%$ of mole fraction there is some overestimation of the maximum catalyst temperature than measured. For Gx-6 experiment since hydrogen concentration and inlet temperature is less compared to $\mathrm{Gx}^{-}$ 8.1 , a comparatively less heat is generated which causes less flow through the recombiner section. The mixture remains for more duration inside the recombiner section; hence efficient conversion of hydrogen takes place. But in $\mathrm{Gx}-$ 8.1 experiment because of high hydrogen concentration the temperature as well as the velocity of mixture remains high in recombiner section. Higher mixture velocity reduces the residual time for mixture remains in the recombiner; hence hydrogen concentration at the outlet is more. Above $8 \% \mathrm{v} / \mathrm{v}$ inlet hydrogen concentration the catalyst surface temperature reaches to $833 \mathrm{~K}$ which is hydrogen autoignition temperature; hence some passive cooling of reaction sheet is required to enable such type of recombiner to use above $8 \% \mathrm{v} / \mathrm{v}$ hydrogen concentration. Research is going on for such type of recombiner design worldwide [9]. Thus detailed analysis gives more useful results as compared to lumped parameters codes which give a single uniform temperature for the catalyst sheet.

\section{Conclusion}

3D CFD analysis of PAR has been carried out for the literature-quoted experimental geometries. The CFD model has been validated with the data from the REKO test facility and Gx test series experiment and given satisfactory prediction. The present analytical model can be used for a plate-type recombiner of any chosen dimensions for predicting its behaviour. The only uncertainty involved in the present analytical model may be in terms of representation of the reaction rate by the chosen Arrhenius equation. These results represent the global behaviour of the recombiner where it

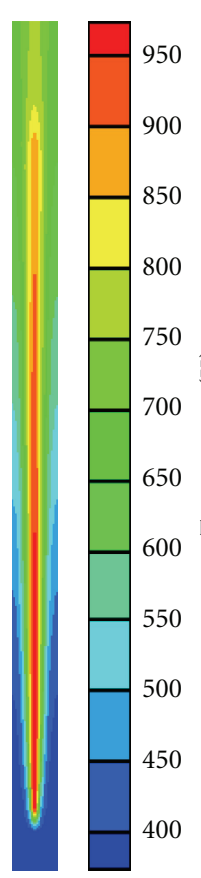

(a)

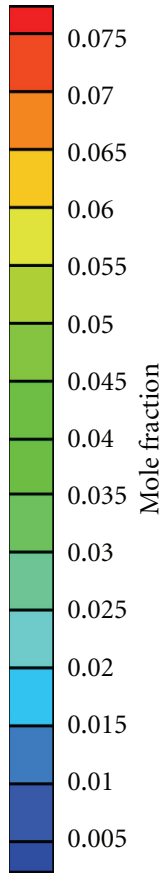

(b)
Figure 16: (a) Temperature contour and (b) hydrogen mole fraction contour zoomed view near catalyst plate second steady state condition $(\mathrm{Gx}-8.1)$.

is assumed that the reaction is occurring over the whole surface. In actual case the reaction is heterogeneous in nature and will be at specific sites. Modeling the detailed reaction mechanism for the recombination process can be considered in future.

\section{Nomenclature}

$\rho:$ Density of mixture $\left(\mathrm{kg} / \mathrm{m}^{3}\right)$

$p$ : Absolute pressure of the mixture $(\mathrm{Pa})$

$T$ : Absolute temperature of the mixture $(\mathrm{K})$

$R_{u}$ : Universal gas constant $(8314 \mathrm{~J} / \mathrm{kmol} \mathrm{K})$

$Y_{i}$ : Mass fraction of the species 
$\mathrm{MW}_{i}:$ Molecular weight of the species

$\left[\mathrm{H}_{2}\right]$ : Molar concentration of hydrogen $\left[\mathrm{Mol} / \mathrm{m}^{3}\right]$

$u$ : $\quad$ Velocity vector

g: $\quad$ Acceleration due to gravity $\left(9.81 \mathrm{~m} / \mathrm{s}^{2}\right)$

$\mu: \quad$ Dynamic viscosity $\left(\mathrm{Ns} / \mathrm{m}^{2}\right)$

$C_{p}: \quad$ Specific heat $(\mathrm{J} / \mathrm{kg}-\mathrm{K})$

$k$ : $\quad$ Thermal conductivity $(\mathrm{W} / \mathrm{m}-\mathrm{K})$

$D: \quad$ Diffusion coefficient $\left(\mathrm{m}^{2} / \mathrm{s}\right)$

$S_{T}$ : Source term in energy equation

$S_{Y i}:$ Source term in species transport equation of the species

$\mathrm{XH}_{2 \text { in }}$ : Mole fraction of hydrogen at recombiner inlet

$T_{\text {in }}$ : Temperature of hydrogen-air mixture at recombiner inlet

$V_{\text {in: }}$ Velocity of hydrogen-air mixture at recombiner inlet.

\section{References}

[1] Mitigation of Hydrogen Hazards in Water Cooled Power Reactors, IAEA, Vienna, IAEA-TECDOC-1196, 2001.

[2] G. Avakian and O. Braillard, "Theoretical model of hydrogen recombiner for a nuclear power plant," in Proceedings of the 7th International Conference on Nuclear Engineering, Tokyo, Japan, 1999.

[3] M. Tahara, H. Oikawa, and K. Arai, "A 3-dimensional fluid dynamic analysis for a passive catalytic recombiner," in Proceedings of the 7th International Conference on Nuclear Engineering, Tokyo, Japan, 1999.

[4] M. Heitsch, "Fluid dynamic analysis of a catalytic recombiner to remove hydrogen," Nuclear Engineering and Design, vol. 201, no. 1, pp. 1-10, 2000.

[5] D. P. Dharwadkar, Analysis of hydrogen distribution in nuclear reactor containment, Ph.D. thesis, Department of Mechanical Engineering, Indian Institute of Technology, Bombay, India, 2007.

[6] D. M. Prabhudharwadkar, K. N. Iyer, and P. Aghalayam, "Numerical simulation of hydrogen mitigation using passive catalytic recombiner," in Proceedings of the 12th International Topical Meeting on Nuclear Reactor Thermal Hydraulics (NURETH-12 '07), Pittsburgh, Pa, USA, 2007.

[7] P. Goyal, Hydrogen transportation and mitigation studies, M.Tech. thesis, Indian Institute of Technology, Kanpur, India, 2001.

[8] P. Drinovac, Experimental studies on catalytic hydrogen recombiners for light water reactors, Ph.D. thesis, RWTH Aachen, Aachen, Germany, 2006.

[9] E. A. Reinecke, I. M. Tragsdorf, and K. Gierling, "Studies on innovative hydrogen recombiners as safety devices in the containments of light water reactors," Nuclear Engineering and Design, vol. 230, no. 1-3, pp. 49-59, 2004.

[10] C. Appel, J. Mantzaras, R. Schaeren, R. Bombach, and A. Inauen, "Catalytic combustion of hydrogen-air mixtures over platinum: validation of hetero/homogeneous chemical reaction schemes," Clean Air, vol. 5, pp. 21-44, 2004.

[11] R. W. Schefer, "Catalyzed combustion of $\mathrm{H}_{2}$ /air mixtures in a flat plate boundary layer: II. Numerical model," Combustion and Flame, vol. 45, pp. 171-190, 1982.

[12] S. V. Patankar, Numerical Heat Transfer and Fluid Flow, Hemisphere, Washington, DC, USA, 1980.
[13] E. A. Reinecke, J. Boehm, P. Drinovac, and S. Struth, "Modelling of catalytic recombiners: comparison of REKO-DIREKT calculations with REKO-3 experiments," in Proceedings of the International Conference Nuclear Energy for New Europe, Bled, Slovenia, 2005. 

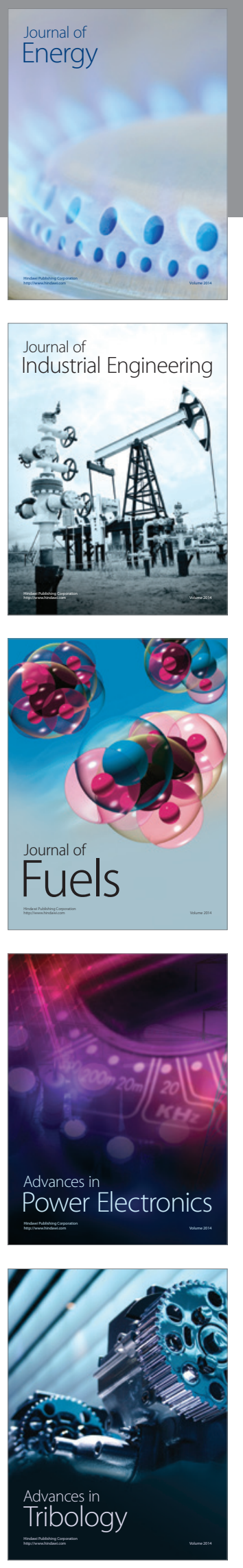
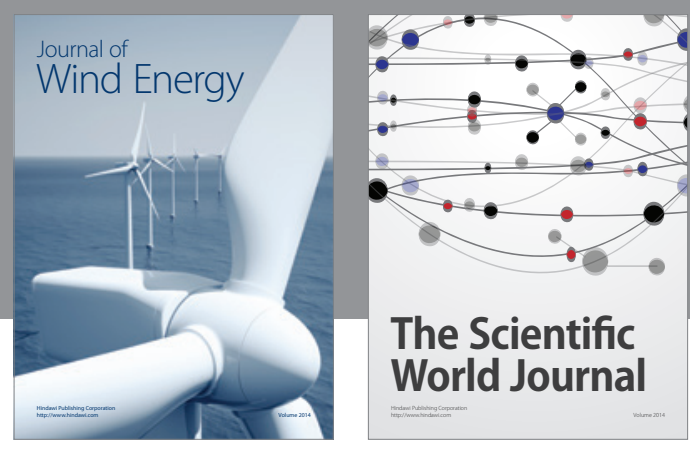

The Scientific World Journal

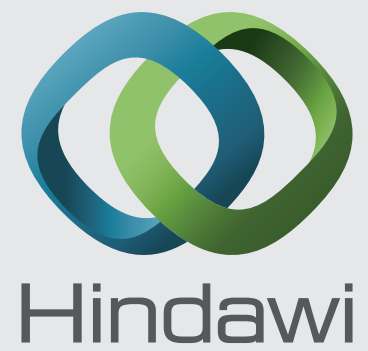

Submit your manuscripts at http://www.hindawi.com
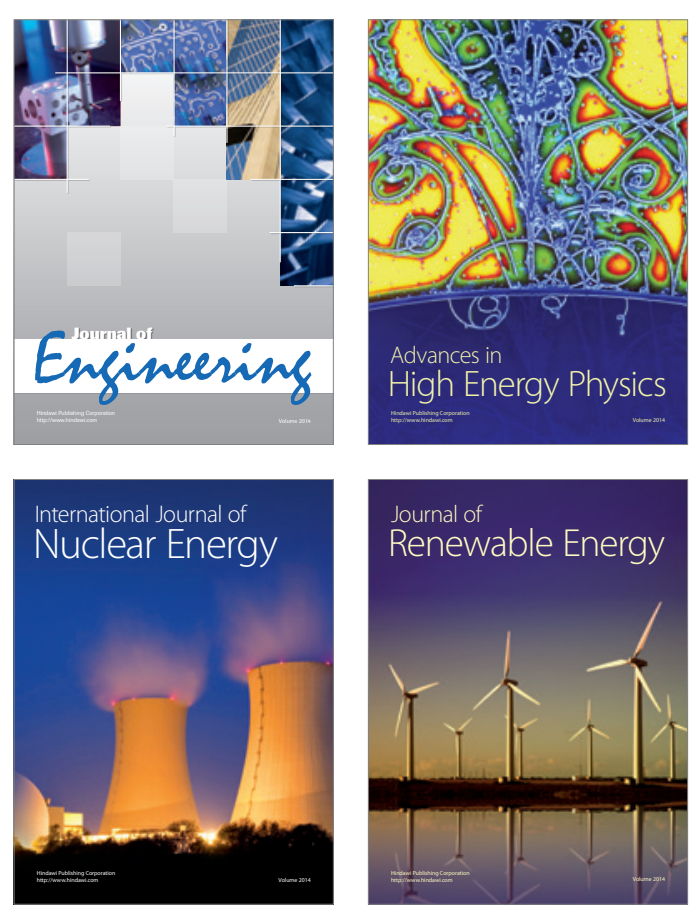

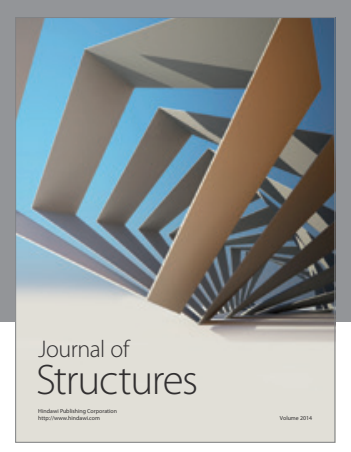

Rotating
Mechinery
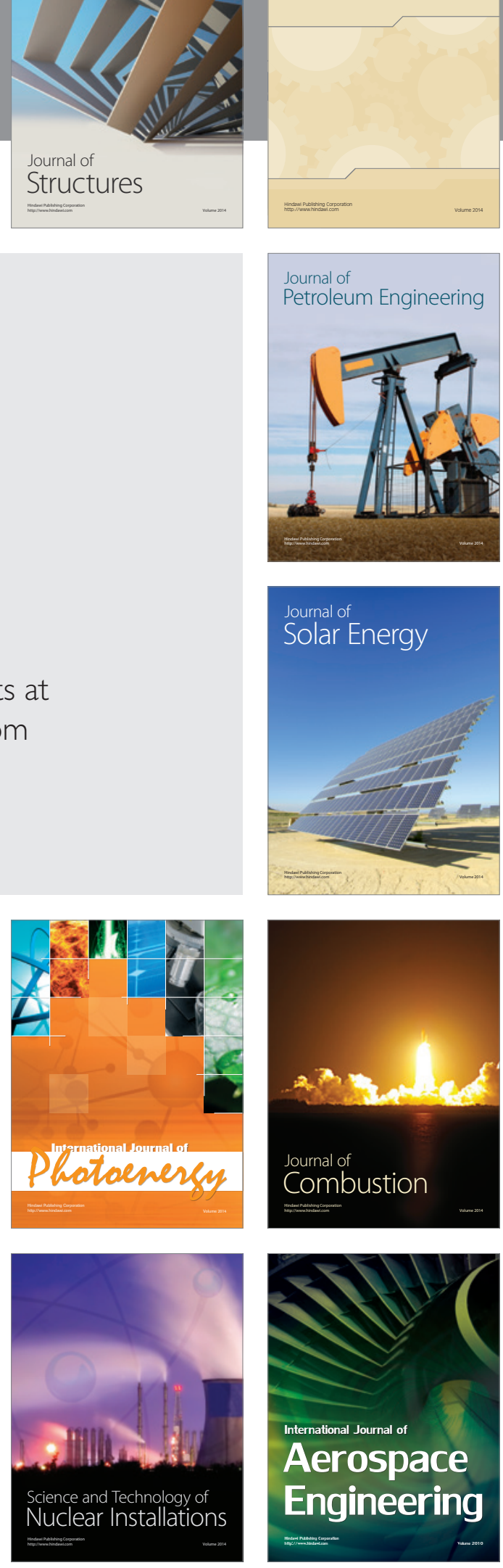